\title{
Ultra-Fast Versus Sustained Cholinergic Transmission: A Variety of Different Mechanisms
}

\author{
Yves Dunant • Victor Bancila $\cdot$ Miguel Cordeiro
}

Received: 6 July 2009 / Accepted: 20 July 2009 / Published online: 24 September 2009

(C) Humana Press 2009

\begin{abstract}
Although synaptic transmission was assumed to use the same mechanisms in the case of different synapses of the central and peripheral nervous system, recent research revealed a great variety of different processes. Time might be a crucial factor to be considered in this diversity. It is recalled that the speed of a chemical reaction is inversely related to affinity. "Time is gained at the expense of sensitivity" as noticed by Bernard Katz (1989). Therefore, synaptic transmission will occur at a high speed only if it is supported by low affinity reactions. In the present work, we compare two examples of ultra-rapid transmission (the Torpedo nerve electroplaque synapse and the rat hippocampus mossy fiber/CA3 synapses), with a cholinergic process operating with high affinity but at a low speed: the release of glutamate elicited by nicotine from mossy fibers of the rat hippocampus.
\end{abstract}

Keywords Hippocampus mossy fibers .

Transmitter release $\cdot$ Nicotine $\cdot$ Membrane potential .

Torpedo electric organ $\cdot$ Vesicular proton gradient

Proceedings of the XIII International Symposium on Cholinergic Mechanisms

Y. Dunant $\cdot$ V. Bancila $\cdot$ M. Cordeiro

Neurosciences Fondamentales, University of Geneva, CMU,

1211 Geneva-4, Switzerland

Y. Dunant $(\varangle)$

Département des neurosciences fondamentales,

Centre Médical Universitaire,

1 rue Michel Servet,

1211 Geneva-4, Switzerland

e-mail: Yves.Dunant@unige.ch

\section{Fast Cholinergic Transmission in the Torpedo Electric Organ}

Torpedoes and some other fish species are provided with electric organs, which are modified neuromuscular junctions. In Torpedo sp., the electric organs contain myriads of cholinergic synapses (approximately $4-6 \times 10^{11}$ /organ), which are typical examples of rapid synapses. Even at $15^{\circ} \mathrm{C}$, the duration of a single electric discharge is only 2 $4 \mathrm{~ms}$. Moreover, the fish can deliver repetitive discharges at frequencies over $100 \mathrm{~Hz}$, for attack or defense. Among the factors ensuring the brevity of synaptic transmission in the electric organ, one can mention the ultra-rapid hydrolysis of acetylcholine (ACh) by acetylcholinesterase, an enzyme that is concentrated in the cleft, like in neuromuscular junctions. All actors directly involved in transmission operate at a high speed but with a low affinity. In the electric organ, nicotinic receptors are activated by submillimolar ACh concentrations; their mean open time is short $(0.6 \mathrm{~ms})$, shorter than in neuromuscular junctions (Sakmann et al. 1985). Similarly, the presynaptic processes which ensure pulse-like transmitter release from nerve endings have to operate at low affinity. Concerning transmission in the Torpedo electric organ, we shall summarize thereafter a series of presynaptic features, which were studied by combining a variety of different approaches (electrophysiology, subcellular fractionation, ultra-rapid freezing, molecular biology, etc. For reviews, see Israël et al. 1979; Dunant 1986, 2000; Dunant and Israël 2000; Dunant et al. 2009).

1. Quanta and subquanta. Transmission is quantal at nerve-electroplaque synapses, with parameters qualitatively and quantitatively similar to those of neuromuscular junctions. The size of evoked and spontaneous 
ACh quanta has been estimated at 6,000-10,000 ACh molecules. A population of "small quanta" or subquanta $(600-1,000$ molecules) is constantly recorded at a low rate under standard conditions, but it becomes prominent at high-release rates and when the energy supply is limited. There is convincing evidence that the "classical" quantum is composed by a mean of ten subquanta. On the other hand, no consistent relation could be found between the quantum size on the one hand and the vesicular ACh content or the diameter of synaptic vesicles on the other hand. For instance, the ACh content of a Torpedo vesicle (100,000-250,000 ACh molecules) would be sufficient for producing 10 25 quanta or 100-250 subquanta.

2. Cytoplasmic ACh, the active compartment. The concentration of ACh in cytoplasmic compartment is surprisingly high $(25-30 \mathrm{mM})$, even in excised electric organ synaptosomes (15-20 mM). ACh synthesis takes place in the cytoplasm, and the newly synthesized transmitter is the first pool released and renewed upon stimulation. Variations in the amount of cytoplasmic $\mathrm{ACh}$ are reflected in characteristic changes of synaptic transmission. Nevertheless, vesicular ACh is also used after prolonged nerve stimulation or mobilized soon after a period of intense activity. Later on, empty vesicles are progressively refilled by the newly synthesized ACh.

3. Fleeting intramembrane particles. An abrupt increase in the density of intramembrane particles (IMPs) accompanies transmission of single impulse in both the $\mathrm{P}$ and $\mathrm{E}$ leaflets of the presynaptic membrane. The duration of the IMP change is very brief, about $3 \mathrm{~ms}$, that is, in the range expected from the duration of the electrophysiological signal. The new IMP population which suddenly appears and disappears has a diameter larger than $10 \mathrm{~nm}$. At $5^{\circ} \mathrm{C}$, both the postsynaptic potential and the IMP change are slowed down and delayed to the same extent. When the experiment is carried out in a high- $\mathrm{Mg}^{2+}$, low- $\mathrm{Ca}^{2+}$ solution, synaptic transmission fails (no transmitter release), and there is no IMP change. These fleeting IMPs also accompany transmitter release in Torpedo and mammalian brain synaptosomes and in reconstituted systems where ACh release is reconstituted, using the mediatophore proteolipid (see below).

4. Fast and slow calcium sequestration into synaptic vesicles. Calcium is taken up by synaptic vesicles via two distinct processes: a high affinity $\mathrm{Ca}^{2+}$-ATPase pump and a $\mathrm{Ca}^{2+} / \mathrm{H}^{+}$antiport. Although diffusion, binding to cytosolic proteins and $\mathrm{Na}^{+} / \mathrm{Ca}^{2+}$ exchange, are probably at work during the rapid phase of presynaptic $\mathrm{Ca}^{2+}$ buffering, the $\mathrm{Ca}^{2+} / \mathrm{H}^{+}$antiport has recently found to be crucial in the process. The $\mathrm{Ca}^{2+} / \mathrm{H}^{+}$antiport displays a very low affinity for $\mathrm{Ca}^{2+}\left(K_{0.5}=217 \mu \mathrm{M}\right.$; maximal activity at $500-600 \mu \mathrm{M})$. As expected, the antiport velocity depends on the $\Delta \mathrm{pH}$ through the vesicular membrane. Upon direct antiport inhibition by strontium ions or indirect inhibition by bafilomycin, the time course of transmitter release is prolonged due to persistence of the $\mathrm{Ca}^{2+}\left(\mathrm{or} \mathrm{Sr}^{2+}\right)$ signal.

5. Exocytosis is delayed and mainly linked to calcium extrusion. At the end of a brief tetanic stimulation, the number of synaptic vesicle does not change but the proportion of vesicles having accumulated calcium transiently increases. Calcium is subsequently cleared from vesicles, probably by exocytosis. Indeed, the density of vesicle openings in the plasma membrane (which does not increase at the very moment of synaptic transmission) very significantly augments during the few minutes following the stimulation train.

6. Mediatophore, sensor and actor in the process of quantal acetylcholine release. The time lag between the presynaptic $\mathrm{Ca}^{2+}$ spark and the onset of the postsynaptic current is incredibly short (about $250 \mu \mathrm{s}$; Llinas et al. 1981; Yazejian et al. 2000). One molecule fulfills all requirements for such an abrupt process: it is the mediatophore, a homo-oligomer of about $220 \mathrm{kDa}$, made up of identical 15 to $16 \mathrm{kDa}$ proteolipid subunits. Mediatophore was isolated and characterized by Israël et al. (1986), using an assay where ACh release from preloaded liposomes was monitored. Cloned and sequenced in Torpedo (Birman et al. 1990) mediatophore turned out to be a member of a proteolipid family, which includes the c-subunit of the membrane sector of V-ATPase. Mediatophore molecules were identified at the presynaptic plasma membrane in the electric organ and neuromuscular junctions. They most probably correspond to the fleeting population of intramembrane particles signaling transmitter release in the presynaptic plasma membrane (Fig. 1A; see above). Mediatophore, reconstituted into liposomes, oocytes, or cell lines enables the preparation to release $\mathrm{ACh}$ in response to a $\mathrm{Ca}^{2+}$ challenge. Release in these reconstituted systems mimics in all aspects what happens in natural synapses, including the production of multimolecular quanta (Fig.1B; Falk-Vairant et al. 1996; Bloc et al. 1999).

\section{Fast Glutamate Transmission at Rat Hippocampus Mossy Fibers/CA3 Synapses}

Electrical stimulation of fibers running from the dentate gyrus to the CA3 area elicits excitatory postsynaptic currents (EPSCs) in CA3 pyramidal cells. These EPSCs 

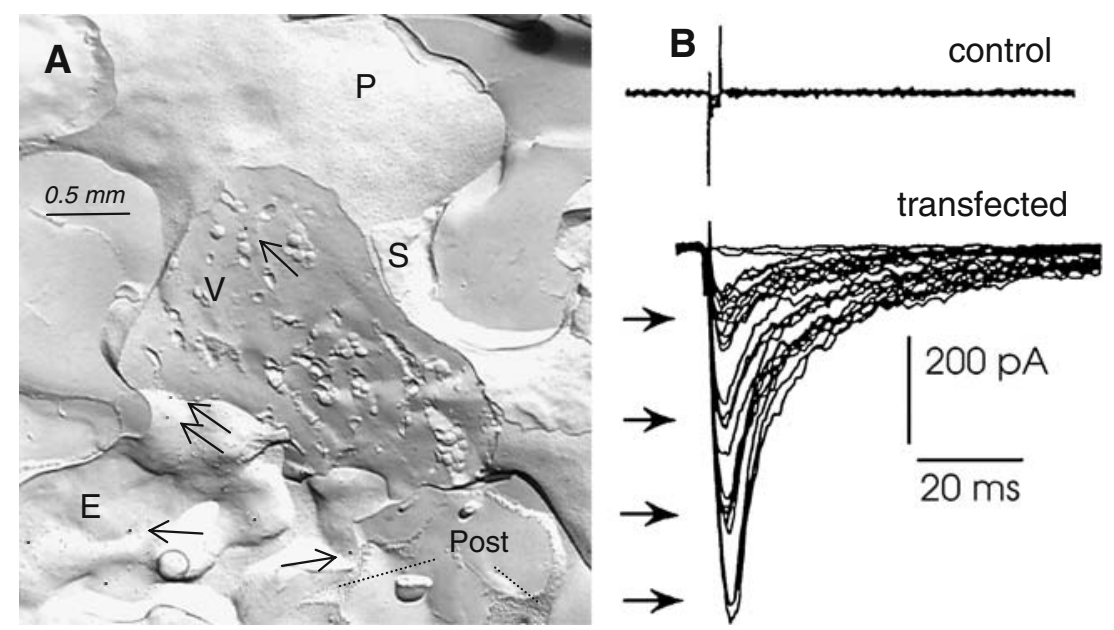

Figure 1 A Nerve-eletroplaque synapse in the Torpedo electric organ, cryofixed during transmitter release. A nerve terminal is transversally fractured, showing synaptic vesicles $(S V)$. The protoplasmic leaflet $(P$, upper area $)$ and the external leaflet $(E$, below) of the presynaptic plasma membrane are exposed. Mediatophore molecules are decorated by gold particles in the replica (arrows) using the labeled fracture technique. Post fragments of the receptor rich postsynaptic plasma membrane; $S$ Schwann cell membrane. B Induction of

have a rapid time course (rise time $0.4 \mathrm{~ms}$; decay time constant $4.8 \mathrm{~ms}$ ). The size of the unitary EPSCs (quantum size) compares well with that of spontaneous miniature EPSCs. The quantum size is quite small $(133 \mathrm{pS})$ in these synapses, corresponding to activation of 14-65 glutamate receptors. For individual mossy fibers, the number of quanta delivered in a single impulse (6-42) meets the quantal ACh release by transfection of mediatophore cDNA. Transmitter release from N18-TG2 cells prefilled with ACh was measured electrophysiologically in real time, by using a Xenopus myoball as a sniffer cell. Control cells were not able to release the transmitter. Cells transfected with the Torpedo mediatophore cDNA released $\mathrm{ACh}$ in a quantal and $\mathrm{Ca}^{2+}$-dependent manner in response to electrical stimuli (see Falk-Vairant et al. 1996)

number (1-27) of postsynaptic spines in contact with a giant terminal (Jonas et al. 1993).

We isolated synaptosomes from rat hippocampus mossy fibers (Fig. 2A) for studying the relationships between the extent of depolarization and the amount of glutamate release. Glutamate release was assayed by a luminescent procedure (Fig. 2B), and the membrane potential by using
Figure 2 A Thin section of a rat hippocampus mossy fiber synaptosome (MFS) showing numerous synaptic vesicles ( $s v)$, as well as by mitochondria $(m)$ and dense core vesicles $(d c v)$. Preand post-synaptic densities ( $p s d$ ) mark active zones of a synapse with a dendritic spine of a pyramidal CA3 neuron. $\mathbf{B}, \mathbf{C}$ Depolarization- and nicotineinduced glutamate release from mossy fiber synaptosomes, as revealed using a continuous luminescence assay for glutamate; after the release run, standard amounts of glutamate were added for calibration

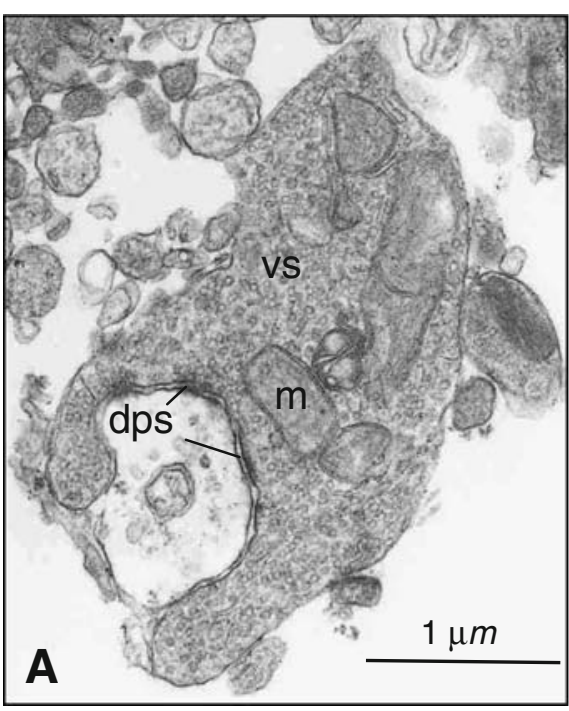

Mossy fiber synaptosomes
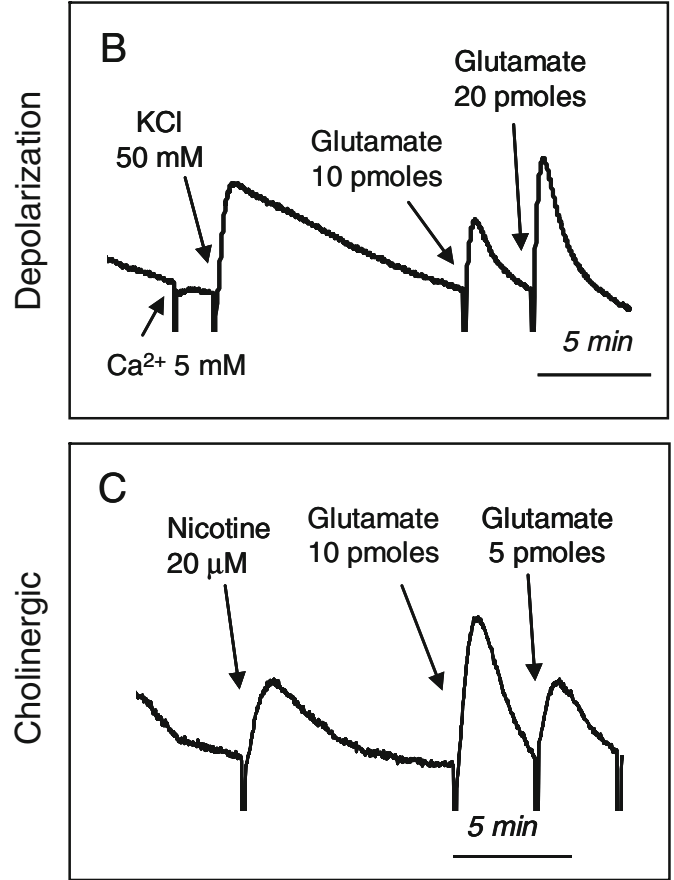

Glutamate release 

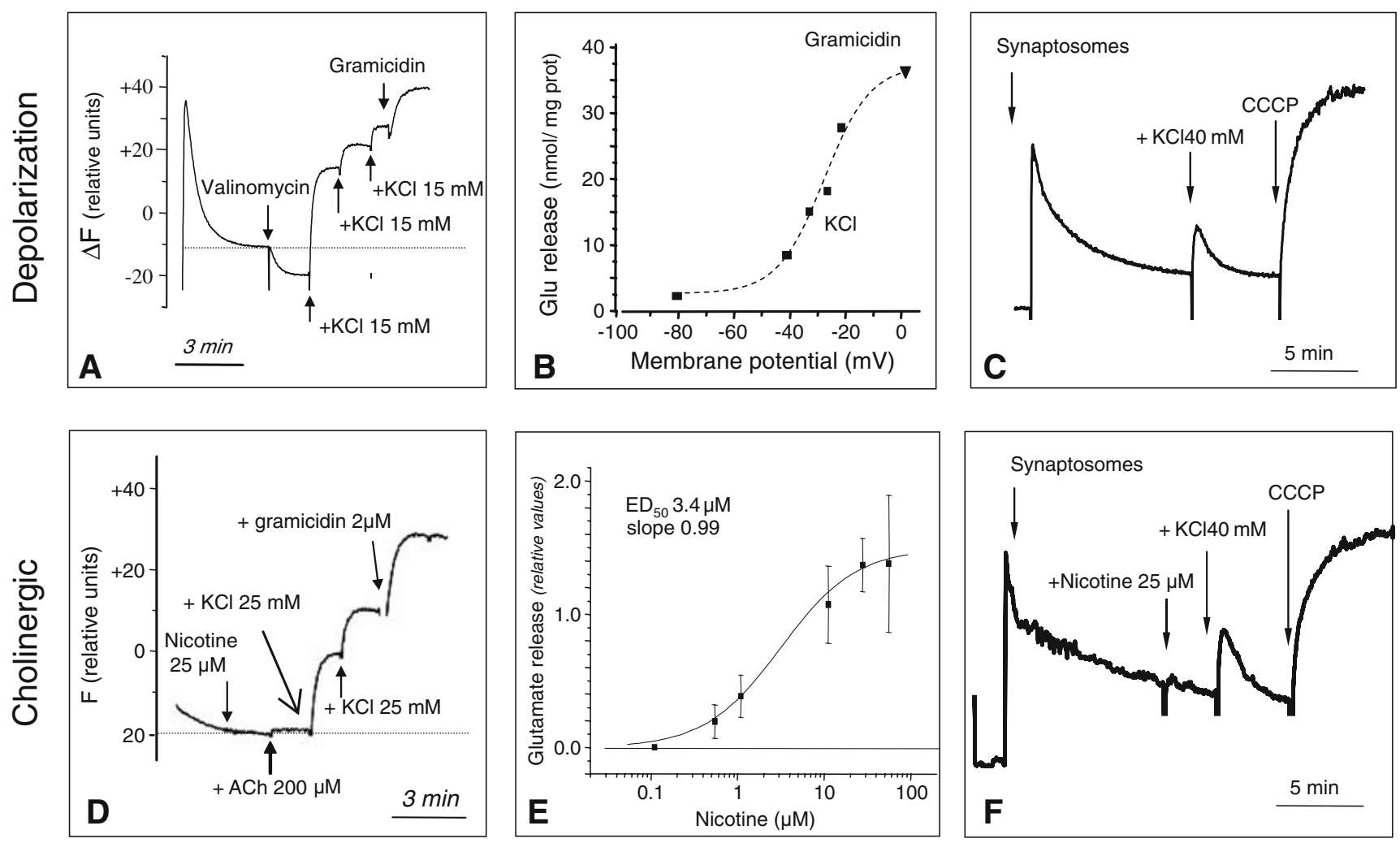

Membrane Potential

(carbocyaninefluorescence)
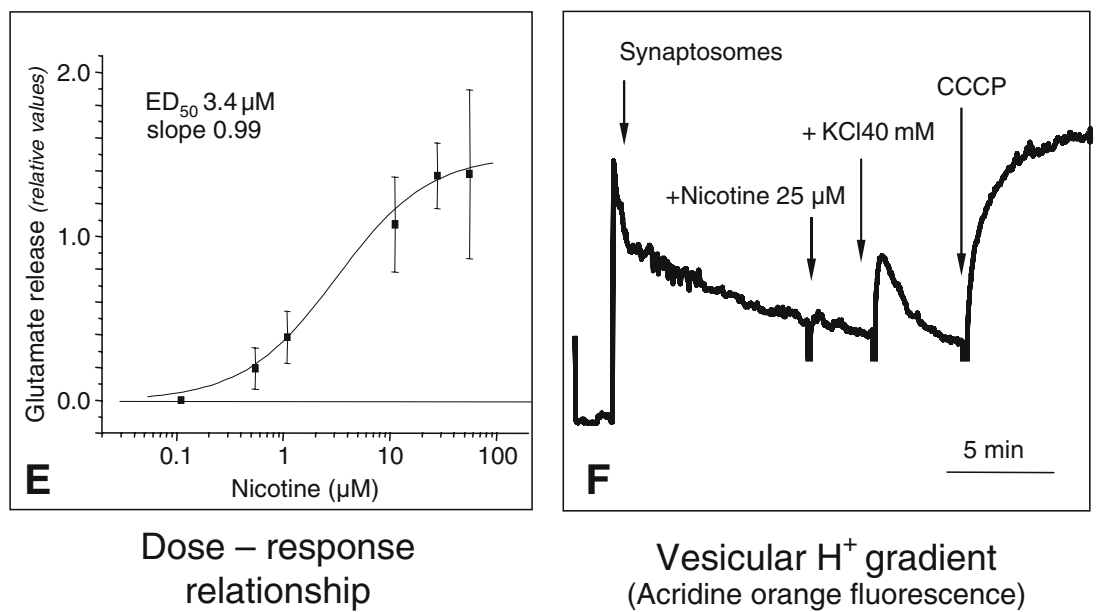

Figure 3 Comparison between mechanisms of depolarizationinduced (upper row) and nicotine-induced (lower row) glutamate release from mossy fiber synaptosomes (MFS). A, D Effects of high$\mathrm{KCl}$, gramicidin, nicotine, and $\mathrm{ACh}$ on the MFS membrane potential, measured using carbocyanin fluorescence; increasing $\mathrm{KCl}$ concentrations depolarize MFSs according to Nernst equation. In contrast,

the fluorescent dye carbocyanine Di-S- $\mathrm{C}_{3(5)}$ (Fig. 3A). The relationships between synaptosome depolarization and glutamate release was established in response to high $\mathrm{KCl}$ and gramicidin challenges. Half-maximal release corresponded to a 52-mV depolarization step (Fig. 3B). We also used acridine orange to assay the proton gradient across the vesicles of mossy fiber synaptic vesicles. $\mathrm{KCl}$-induced glutamate release was accompanied by transient dissipation of the vesicular proton gradient (Fig. 3C), as observed using brain cortex synaptosomes (Zoccarato et al. 1999).

\section{High-Affinity Activation of Glutamate Release from Mossy Fibers by Nicotine}

Electrophysiological experiments showed that nicotine, at a low concentration, induces quantal glutamate release from mossy fibers (MF). Curiously, the effect is delayed (about $1 \mathrm{~min})$; it requires $\mathrm{Ca}^{2+}$ at the time of nicotine application but not at the time of release (Sharma and Vijayaraghavan 2003; Sharma et al. 2008). nicotine provokes transmitter release without affecting the membrane potential. B Depolarization-release relationships of MFSs in vitro. E Dose-response relationship for nicotine-induced glutamate release from MFSs. C, F High KCL induces a transient dissipation of the MSF vesicular proton gradient, while nicotine releases glutamate without affecting the vesicular proton gradient

We also used nicotine to elicit glutamate release from mossy fiber synaptosomes. Figure $3 \mathrm{C}$ shows that micromolar doses of nicotine are efficient. The $\mathrm{EC}_{50}$ value for this effect is $3.14 \mu \mathrm{M}$ (Fig. 3E). Surprisingly, nicotine does not cause any significant depolarization of the synaptosomes (Fig. 3D) or any dissipation of the vesicular proton gradient (Fig. 3F). Therefore, the mechanism of nicotine-induced glutamate release does not pass through depolarizationinduced voltage-dependent calcium channels but through slow processes, probably involving calcium-induced calcium release.

\section{Conclusion}

It was generally assumed that nicotinic receptors (and ionchannel receptors of the same family) were always involved in rapid synaptic transmission (effects arising within milliseconds), while muscarinic receptors (and others "metabotropic" receptors) would play a role in slower communication processes (seconds or even minutes). This view must be 
questioned since nicotinic-activation of transmitter release in the CNS does not seem to operate in a flash-like manner, like nicotinic neuromuscular transmission. Rather, some CNS nicotinic receptors share several characteristics with "metabotropic" receptors: high-affinity activation, relatively slow and prolonged cellular effects, involvement of second messengers, calcium-induced calcium release and other similar processes. Therefore, the central and peripheral nervous systems display an amazing variety of different cholinergic mechanisms.

\section{References}

Birman, S., Meunier, F. M., Lesbats, B., LeCaer, J. P., Rossier, J., \& Israël, M. (1990). A $15 \mathrm{kD}$ proteolipid found in mediatophore preparations from Torpedo presents high sequence homology with the bovine chromaffin granule protonophore. FEBS Lett, 261, 303-306.

Bloc, A., Bugnard, E., Dunant, Y., Falk-Vairant, J., Israël, M., Loctin, F., et al. (1999). Acetylcholine synthesis and quantal release reconstituted by transfection of mediatophore and choline acetyltransferase cDNAs. Eur J Neurosci, 11, 1523-1534.

Dunant, Y. (1986). On the mechanism of acetylcholine release. Progress in Neurobiology, 26, 55-92.

Dunant, Y. (2000). Quantal acetylcholine release : Vesicle fusion or intramembrane particles? Microscopy Research and Technique, $49,38-46$.

Dunant, Y., \& Israël, M. (2000). Neurotransmitter release in rapid synapses. Biochimie, 82, 289-302.

Dunant, Y., Cordeiro, J. M., \& Goncalves, P. P. (2009). Exocytosis, mediatophore, and vesicular $\mathrm{Ca}_{2}^{+} / \mathrm{H}^{+}$antiport in rapid neuro- transmission. Annals of the New York Academy of Sciences, 1152, $100-112$.

Falk-Vairant, J., Corrèges, P., Eder-Colli, L., Salem, N., Roulet, E., Bloc, A., et al. (1996). Quantal acetylcholine release induced by mediatophore transfection. Proc Natl Acad Sci USA, 93, 5203-5207.

Israël, M., Dunant, Y., \& Manaranche, R. (1979). The present status of the vesicular hypothesis. Progress Neurobiology, 13, 237-275.

Israël, M., Morel, N., Lesbats, B., Birman, S., \& Manaranche, R. (1986). Purification of a presynaptic membrane protein that mediates a calcium-dependent translocation of acetylcholine. Proc Natl Acad Sci U S A, 83, 9226-9230.

Jonas, P., Major, G., \& Sakmann, B. (1993). Quantal components of unitary EPSCs at the mossy fibre synapse on CA3 pyramidal cells of rat hippocampus. $J$ Physiol, 472, 615-663.

Katz, B. (1989). Looking back at the neuromuscular junction. In L. C. Sellin, R. Libelius \& S. Thesleff (Eds.), Neuromuscular junction (pp. 3-9). Amsterdam: Elsevier.

Llinas, R., Steinberg, I. Z., \& Walton, K. (1981). Relationship between presynaptic calcium current and postsynaptic potential in squid giant synapse. Biophys $J, 33,323-352$.

Sakmann, B., Methfessel, C., Mishina, M., Takahashi, T., Takai, T., Kurasaki, M., et al. (1985). Role of acetylcholine receptor subunits in gating of the channel. Nature, 318, 538-543.

Sharma, G., \& Vijayaraghavan, S. (2003). Modulation of presynaptic store calcium induces release of glutamate and postsynaptic firing. Neuron, 38, 929-939.

Sharma, G., Grybko, M., \& Vijayaraghavan, S. (2008). Action potential-independent and nicotinic receptor-mediated concerted release of multiple quanta at hippocampal CA3-mossy fiber synapses. J Neurosci, 28, 2563-2575.

Yazejian, B., Sun, X. P., \& Grinnell, A. D. (2000). Tracking presynaptic $\mathrm{Ca} 2+$ dynamics during neurotransmitter release with Ca2+-activated K+channels. Nat Neurosci, 3, 566-571.

Zoccarato, F., Cavallini, L., \& Alexandre, A. (1999). The pH-sensitive dye acridine orange as a tool to monitor exocytosis/endocytosis in synaptosomes. $J$ Neurochem, 72, 625-633. 